\title{
Supersymmetric Monopole Quantum Mechanics on Sphere
}

\author{
Soon-Tae Hong* \\ Department of Science Education, Ewha Womans University, Seoul 120-750 Korea \\ Joohan Leđ \\ Department of Physics, University of Seoul, Seoul 130-743 Korea \\ Tae Hoon Lef \\ Department of Physics, Soongsil University, Seoul 156-743 Korea \\ Phillial $\mathrm{Oh} \S$ \\ Department of Physics and Institute of Basic Science, \\ Sungkyunkwan University, Suwon 440-746 Korea
}

(Dated: August 21, 2018)

\begin{abstract}
We study $N=2$ supersymmetric quantum mechanics of a charged particle on sphere in the background of Dirac magnetic monopole. We adopt $C P(1)$ model approach in which the monopole interaction is free of singularity. It turns out that this approach admits a compact $N=2$ superspace formulation. In order to exploit manifest $U(1)$ covariance in the superspace formalism, we introduce a gauged chiral superfield which is annihilated by the gauge covariant superderivative instead of the usual superderivative. We carry out the Dirac quantization of the resulting system and compute the quantum mechanical spectrum. We obtain the condition for the spontaneous breaking of supersymmetry explicitly in terms of the monopole charge and a parameter which characterizes the operator ordering ambiguity. We find that the supersymmetry is spontaneously broken unless a certain combination of these quantities satisfies some quantization condition.
\end{abstract}

PACS numbers: 11.30.Pb, 11.30.Qc, 14.80.Hv

Keywords: supersymmetric quantum mechanics; magnetic monopole; operator ordering; supersymmetry breaking

\section{INTRODUCTION}

Quantum mechanics in the background of Dirac magnetic monopole [1] exhibits many interesting features such as quantization of the electric charge, modified orbital angular momentum and hidden conformal symmetries associated with the time reparametrization invariance 2, 3. . The supersymmetric magnetic monopole quantum mechanics has attracted a great deal of attention recently due to the existence of hidden superconformal symmetry [4] and in relation with superconformal mechanics [5]. In this paper, we study $N=2$ supersymmetric quantum mechanics $[\underline{6}]$ of a charged particle on a sphere in the background of Dirac magnetic monopole by using the chiral superfield formalism.

Our motivation for considering this system is twofold. One is that the supersymmetric quantum mechanics on general target manifold (regardless of the presence of magnetic monopole) is interesting in itself and its study revealed many important aspects of supersymmetry [7]. The other concerns with the number of supersymmetries allowed when the magnetic monopole interaction is present. It is well known that the chiral $N=2$ superfield formulation of supersymmetric quantum mechanics on $S^{2}$ is possible because of its Kähler structure 8, 9]. However, as far as the magnetic monopole interaction is concerned, most of the previous work dealt with $N=1$ superfields in $\mathbf{R}^{3}$ [10]. Later, it was found that this system admits another supersymmetry and its relation with the constrained dynamics on a sphere was discussed 11]. More recently, $N=2$ supersymmetric quantum mechanics of a charge-monopole system confined to $S^{2}$ has been investigated in terms of unconstrained variables [12. Utilizing the shape-invariance of the system, they obtained the energy spectrum and a complete set of energy eigenstates [12] with a particular choice of the operator ordering [13].

\footnotetext{
*Electronic address: soonhong@ewha.ac.kr

${ }^{\dagger}$ Electronic address: joohan@kerr.uos.ac.kr

${ }^{\ddagger}$ Electronic address: thlee@ssu.ac.kr

$\S$ Electronic address: ploh@newton.skku.ac.kr
} 
In this work, we adopt $C P(1)$ model approach 14] where the dynamical variables take the value on $S^{3}$, but the dynamics is reduced to $S^{2}$ by imposing $U(1)$ gauge symmetry. This has the merit that the quantum mechanical Lagrangian of magnetic monopole is free of singularity [15] and one does not have to deal with the multi-valued action [16]. It also has the advantage that the rotational generators are well realized. We find that this approach allow a compact $N=2$ superfield formulation of the system including the monopole interaction. In order to exploit the $U$ (1) gauge covariance we introduce gauged chiral superfield which is annihilated by the gauge covariant superderivative, in which the time derivative in the usual superderivative is replaced with the gauge covariant one. This gauge covariant superderivative still satisfies the usual important property that it commutes with the supersymmetry generators (see Eqs (2.1), (2.5) and (2.7)).

In quantizing the system there appears a parameter associated with the choice of operator ordering in defining the basic commutation relations. We study how physical quantities such as energy and angular momentum depend on this parameter. We obtain the exact quantum mechanical energy spectrum and discuss the possibility of spontaneous breaking of supersymmetry in terms of the monopole charge and the ordering parameter.

Let us briefly recall the bosonic $C P(1)$ model. The Lagrangian is given by

$$
L_{0}=2\left|D_{t} z_{i}\right|^{2}
$$

with $D_{t}=\partial_{t}-i a$, where $a$ is the auxiliary field. Impose the condition $\bar{z} \cdot z \equiv \sum_{i=1}^{2}\left|z_{i}\right|^{2}=1$ with $z=\left(\begin{array}{c}z_{1} \\ z_{2}\end{array}\right)$. Due to the $U(1)$ invariance, the dynamics is reduced from $S^{3}$ to $S^{2}$, which is the $C P(1)$ model. Eliminating the auxiliary field $a$ by using the equation of motion, we obtain

$$
L_{1}=2|\dot{z}+(\dot{\bar{z}} \cdot z) z|^{2}
$$

where the overdot denotes the time derivative. The magnetic monopole background interaction is given by

$$
L_{2}=i g(\bar{z} \cdot \dot{z}-\dot{\bar{z}} \cdot z)
$$

where $g$ is the magnetic monopole charge ${ }^{1}$. Observe that the Lagrangian $L_{1}$ is invariant under the $U(1)$ gauge symmetry generated by $z \rightarrow e^{i \Lambda(t)} z$, whereas $L_{2}$ changes by a total time derivative. They are free of singularities. The singular Lagrangian emerges through the Hopf fibration $\vec{x}=\bar{z} \vec{\sigma} z$ [17] and the introduction of local coordinates $z_{1}=1 / \sqrt{1+|\xi|^{2}}, z_{2}=\xi / \sqrt{1+|\xi|^{2}}$. The stereographic projection $\xi=\tan \frac{\theta}{2} e^{i \phi}$ produces the monopole interaction which is singular along the negative $z$-axis. The choice $\xi=\cot \frac{\theta}{2} e^{i \phi}$ gives singularity along the positive $z$-axis. Both choices yield the standard kinetic Lagrangian $L_{1}=\frac{1}{2} \dot{\vec{x}} \cdot \dot{\vec{x}}$.

This paper is organized as follows. In section 2, we define gauge covariant superderivatives and gauged chiral superfield. We then construct $N=2$ supersymmetric monopole Lagrangian. In section 3, we quantize the system via Dirac quantization method and discuss operator ordering ambiguity. In section 4, we compute the energy spectrum and analyze the spontaneous supersymmetry breaking phenomena. Section 5 includes summary and discussions.

\section{II. $N=2$ SUPERSYMMETRIC MONOPOLE LAGRANGIAN}

We present our $N=2$ supersymmetric Lagrangian in a $U(1)$ covariant manner. First, we introduce superspace $(t, \theta, \bar{\theta})$ and define the gauge covariant superderivatives as

$$
\begin{aligned}
D & =\partial_{\theta}-i \bar{\theta} D_{t}, \\
\bar{D} & =\partial_{\bar{\theta}}-i \theta D_{t},
\end{aligned}
$$

where $D_{t}$ denotes $U(1)$ covariant derivative,

$$
\begin{aligned}
& D_{t} z=\left(\partial_{t}-i a\right) z, \\
& D_{t} \bar{z}=\left(\partial_{t}+i a\right) \bar{z} .
\end{aligned}
$$

for some real field $a$, which we will specify shortly. Note that the covariant superderivatives $D$ and $\bar{D}$ satisfy

$$
D^{2}=\bar{D}^{2}=0, \quad[D, \bar{D}]_{+}=-2 i D_{t}
$$

\footnotetext{
${ }^{1}$ Here, we set the electric charge $e=-1$.
} 
$N=2$ gauged chiral superfield, $\Phi$, is defined as usual by imposing the condition $\bar{D} \Phi=0$. Thus, we get

$$
\Phi=z+\theta \psi-i \theta \bar{\theta} D_{t} z
$$

Supersymmetry generators are similarly modified to

$$
\begin{aligned}
& Q=\partial_{\theta}+i \bar{\theta} D_{t}, \\
& \bar{Q}=\partial_{\bar{\theta}}+i \theta D_{t},
\end{aligned}
$$

which satisfy

$$
[Q, \bar{Q}]_{+}=2 i D_{t}
$$

and fulfill the relations

$$
Q^{2}=\bar{Q}^{2}=0, \quad[D, Q]_{+}=[D, \bar{Q}]_{+}=[\bar{D}, Q]_{+}=[\bar{D}, \bar{Q}]_{+}=0 .
$$

Transformation rules of the chiral field components are obtained by applying $Q$ and $\bar{Q}$ to the superfields. From

$$
\begin{aligned}
Q \Phi & =\delta z-\theta \delta \psi-i \theta \bar{\theta} \delta\left(D_{t} z\right) \\
& =\psi-i \theta \bar{\theta} D_{t} \psi
\end{aligned}
$$

we get

$$
\delta z=\psi, \quad \delta \psi=0, \quad \delta\left(D_{t} z\right)=D_{t} \psi
$$

Similarly, from

$$
\begin{aligned}
Q \bar{\Phi} & =\delta \bar{z}+\bar{\theta} \delta \bar{\psi}+i \theta \bar{\theta} \delta\left(D_{t} \bar{z}\right) \\
& =2 i \bar{\theta} D_{t} \bar{z}
\end{aligned}
$$

we find

$$
\delta \bar{z}=0, \quad \delta \bar{\psi}=2 i D_{t} \bar{z}, \quad \delta\left(D_{t} \bar{z}\right)=0 .
$$

Note that both of these transformation rules require for consistency that

$$
\delta a=0 .
$$

Calculation for $\bar{Q}$ leads to the similar transformation rules for the component fields and the consistency condition, $\bar{\delta} a=0$. We summarize the supertransformation of the fields,

$$
\begin{aligned}
& \delta z=\psi, \delta \bar{z}=0, \quad \delta \psi=0, \quad \delta \bar{\psi}=2 i D_{t} \bar{z}, \\
& \bar{\delta} z=0, \quad \bar{\delta} \bar{z}=\bar{\psi}, \quad \bar{\delta} \psi=2 i D_{t} z, \bar{\delta} \bar{\psi}=0 .
\end{aligned}
$$

Our supersymmetric action is then proposed by

$$
L=\int \mathrm{d} \bar{\theta} \mathrm{d} \theta\left(\frac{1}{2} \overline{D \Phi} \cdot D \Phi\right)-2 g a
$$

with the superconstraint

$$
\bar{\Phi} \cdot \Phi-1=0 .
$$

This superfield constraint incorporates the familiar constraints [14] $\bar{z} \cdot z-1=0, \bar{z} \cdot \psi=0=\bar{\psi} \cdot z$, and determines the field $a$,

$$
a=-\frac{i}{2}(\bar{z} \cdot \dot{z}-\dot{\bar{z}} \cdot z)-\frac{1}{2} \bar{\psi} \cdot \psi
$$


It is important to note that the field $a$ obtained above is indeed invariant under the supersymmetry transformations to yield Eq. (2.12). This is what makes our whole construction consistent. After performing the $\theta$ and $\bar{\theta}$ integrations (and using the constraints), we obtain

$$
L=2\left|D_{t} z\right|^{2}+\frac{i}{2}\left(\bar{\psi} \cdot D_{t} \psi-D_{t} \bar{\psi} \cdot \psi\right)-2 g a
$$

Substituting Eq. (2.16) into Eq. (2.17), we can express the $N=2$ supersymmetric Lagrangian in the following form,

$$
L=2|\dot{z}-(\bar{z} \cdot \dot{z}) z|^{2}+\frac{i}{2}(\bar{\psi} \cdot \dot{\psi}-\dot{\bar{\psi}} \cdot \psi)-\frac{i}{2}(\bar{z} \cdot \dot{z}-\dot{\bar{z}} \cdot z) \bar{\psi} \cdot \psi+i g(\bar{z} \cdot \dot{z}-\dot{\bar{z}} \cdot z-i \bar{\psi} \cdot \psi)
$$

The fact that the Lagrangian is supersymmetric should be clear for it was written in terms of superfields. At the component field level it can be most easily verified using Eq. (2.17). It is interesting to note that the supersymmetric magnetic monopole interaction term in Eq. (2.18) is given by the field $a$ itself. In this model $U(1)$ gauge transformation of the monopole potential is realized by the local $U(1)$ symmetry which is responsible for the reduction from $S^{3} \rightarrow S^{2}$. In Appendix A, we give the full equations of motion.

\section{DIRAC QUANTIZATION AND OPERATOR ORDERING}

In this section, we perform the canonical quantization of the system. We define the momenta $p$ and $\bar{p}$ conjugate to the fields $z$ and $\bar{z}$, respectively by $^{2}$

$$
p=2 D_{t} \bar{z}+\frac{i}{2}(\bar{\psi} \cdot \psi+2 g) \bar{z}, \quad \bar{p}=2 D_{t} z-\frac{i}{2}(\bar{\psi} \cdot \psi+2 g) z .
$$

The Hamiltonian is obtained as

$$
H=2\left|D_{t} z\right|^{2}-\frac{1}{2}(\bar{\psi} \cdot \psi)^{2}-g \bar{\psi} \cdot \psi,
$$

supplemented by the following four second class constraints

$$
C_{1}=\bar{z} \cdot z-1, \quad C_{2}=p \cdot z+\bar{z} \cdot \bar{p}, \quad C_{3}=\bar{z} \cdot \psi, \quad C_{4}=\bar{\psi} \cdot z .
$$

There is also a first class constraint given by

$$
C_{0}=-i(\bar{z} \cdot \bar{p}-p \cdot z)-\bar{\psi} \cdot \psi+2 g,
$$

which generates the $U(1)$ transformation. We first define the Poisson brackets via

$$
\left\{z_{i}, p_{j}\right\}=\left\{\bar{z}_{i}, \bar{p}_{j}\right\}=\delta_{i j}, \quad\left\{\bar{\psi}_{i}, \psi_{j}\right\}_{+}=-i \delta_{i j}
$$

with the remaining brackets being zero. We use the Dirac brackets given by

$$
\{A, B\}_{D}=\{A, B\}-\left\{A, C_{a}\right\} \Theta^{a b}\left\{C_{b}, B\right\},
$$

where $\Theta^{a b}$ is the inverse matrix of $\Theta_{a b}=\left\{C_{a}, C_{b}\right\}$. After some computation and quantizing the Dirac brackets by replacing $\{A, B\}_{D} \rightarrow-i[A, B]$, we obtain

$$
\begin{array}{ll}
{\left[p_{i}, z_{j}\right]=-i \delta_{i j}+\frac{i}{2} \bar{z}_{i} z_{j},} & {\left[p_{i}, \bar{z}_{j}\right]=\frac{i}{2} \bar{z}_{i} \bar{z}_{j},} \\
{\left[p_{i}, p_{j}\right]=\frac{i}{2}\left(p_{i} \bar{z}_{j}-p_{j} \bar{z}_{i}\right),} & {\left[\bar{p}_{i}, p_{j}\right]=\frac{i}{2}\left(\bar{z}_{j} \bar{p}_{i}-z_{i} p_{j}\right)-\alpha \psi_{i} \bar{\psi}_{j}+\beta \bar{\psi}_{j} \psi_{i},} \\
{\left[\bar{\psi}_{i}, \psi_{j}\right]_{+}=\delta_{i j}-\bar{z}_{i} z_{j},} & {\left[p_{i}, \bar{\psi}_{j}\right]=i \bar{\psi}_{i} \bar{z}_{j},}
\end{array}
$$

with $\alpha+\beta=1$. The above brackets are supplemented by their Hermitian conjugates, and remaining commutators are zero. Note that the brackets in the first and third lines of Eq. (3.7) have no operator ordering ambiguity. In

\footnotetext{
${ }^{2}$ Here, we have chosen a specific ordering of the quantum mechanical operators $p$ and $\bar{p}$. Especially we take $D_{t} z=\dot{z}-i z a$ and its conjugate $D_{t} \bar{z}=\dot{\bar{z}}+i a \bar{z}$.
} 
the second line, the ordering of the first bracket is fixed by the anti-symmetry property, while ordering in the second bracket is chosen by the condition that the variables $\left(z_{i}, \bar{z}_{i}, \psi_{i}, \bar{\psi}_{i}, p_{i}, \bar{p}_{i}\right)$ commute with the second class constraint, $C_{2}$, ordered as $p \cdot z+\bar{z} \cdot \bar{p}=0$. Similar ordering choice appeared before in the bosonic $C P(1)$ model $[18$. Note that this does not fix the operator ordering completely in the fermionic case, and we still have undetermined $\alpha$ and $\beta$ in Eq. (3.7).

We then compute the Noether charge associated with phase symmetry of the fermionic variables

$$
N_{F}=\bar{\psi} \cdot \psi
$$

which turns out to be the fermion number operator. In fact, using the constraints one can derive the following result.

$$
(\bar{\psi} \cdot \psi)^{2}=\bar{\psi} \cdot \psi
$$

therefore $N_{F}=0$ or 1 . The supersymmetry charges are given by

$$
Q=p \cdot \psi, \quad \bar{Q}=\bar{\psi} \cdot \bar{p}
$$

Note that the supercharges have no ordering ambiguity. One can easily check that $[\bar{\psi} \cdot \psi, Q]=-Q,[\bar{\psi} \cdot \psi, \bar{Q}]=\bar{Q}$. Thus, $Q$ and $\bar{Q}$ play the role of lowering and raising operator of the fermion number.

The global $\mathrm{SU}(2)$ rotations are generated by

$$
\left(\begin{array}{l}
z_{1} \\
z_{2}
\end{array}\right) \rightarrow e^{-\frac{i}{2} w^{a} \sigma^{a}}\left(\begin{array}{l}
z_{1} \\
z_{2}
\end{array}\right),
$$

whose operator-ordered conserved charge is given by

$$
K_{a}=\frac{i}{2} \bar{z} \sigma_{a} \bar{p}-\frac{i}{2} p \sigma_{a} z+\gamma \bar{z} \sigma_{a} z+\frac{1}{2} \bar{\psi} \sigma_{a} \psi
$$

Here we have added the third term associated with the operator ordering ambiguity. After some computation, we find that $K_{a}$ 's generate the $\mathrm{SU}(2)$ algebra

$$
\left[K_{a}, K_{b}\right]=i \epsilon_{a b c} K_{c}
$$

provided the following conditions are satisfied

$$
\alpha=\frac{1}{2}(1+4 \gamma), \beta=\frac{1}{2}(1-4 \gamma)
$$

Note that the angular momentum algebra Eq. (3.13) is satisfied independent of the value $\gamma$. In Appendix B, we give various commutation relations of $K_{a}$ 's with other operators and express them in terms of space unit vector $\vec{x}=\bar{z} \vec{\sigma} z$.

\section{ENERGY SPECTRUM AND SUPERSYMMETRY BREAKING}

The quantum mechanical Hamiltonian is defined by

$$
\begin{aligned}
H_{q} & \equiv \frac{1}{2}[Q, \bar{Q}]_{+} \\
& =\frac{1}{2} p \cdot \bar{p}-\frac{1}{2} g^{2}-\frac{1}{2}(g+2 \gamma) \bar{\psi} \cdot \psi-\frac{1}{8}(\bar{\psi} \cdot \psi)^{2},
\end{aligned}
$$

which differs from the classical Hamiltonian Eq. (3.2) by operator ordering. This Hamiltonian can be expressed in terms of $K_{a}$ operators as

$$
H_{q}=\frac{1}{2}\left[K^{2}-(g+\gamma)(g+\gamma+1)\right]
$$

Thus, we obtain the energy spectrum as follows

$$
E=\frac{1}{2}[k(k+1)-\tilde{g}(\tilde{g}+1)],
$$




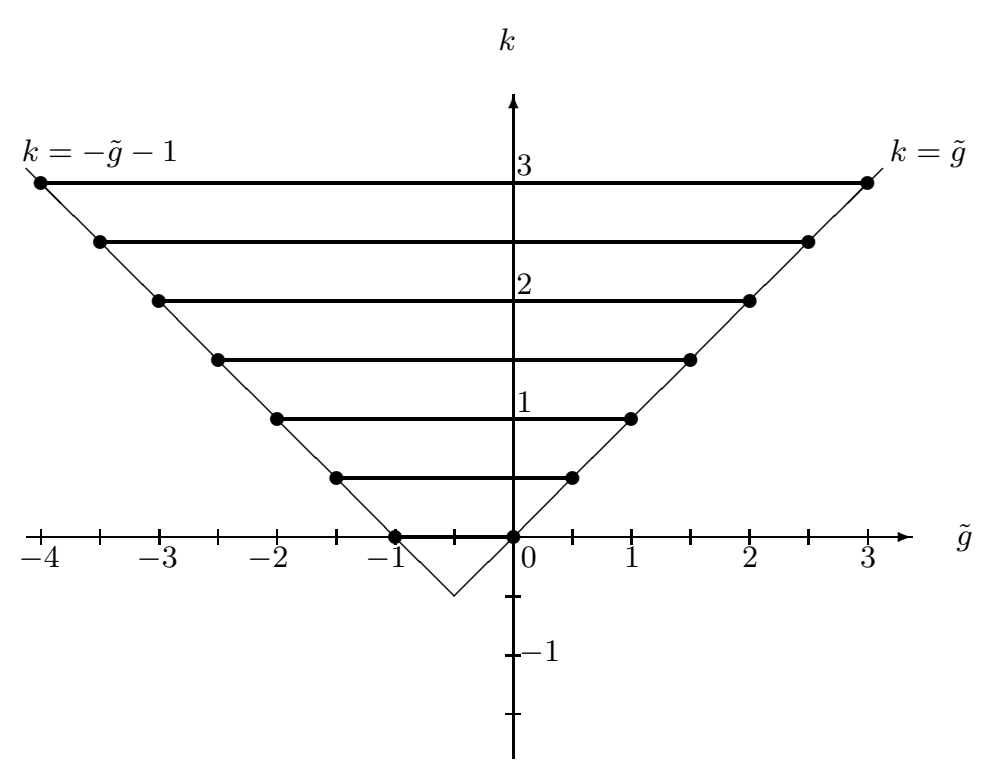

FIG. 1: Diagram for $k$ versus $\tilde{g}$.

where $k=0, \frac{1}{2}, 1, \cdots$ is the angular quantum number associated with $K_{a}$ operator and $\tilde{g}=g+\gamma$. Some comments are in order at this point. The energy $E$ must be positive definite because of the first equation of (4.1). Moreover, the spectrum is obtained by exploiting the rotational invariance. In the case of $\gamma=0$, the method of raising and lowering operators can be used to construct the energy eigenvalues [12]. The Hamiltonian commutes with the fermion number $N_{F}$ and $K_{a}$ 's and thus the spectrum has a $2(2 k+1)$-fold degeneracy.

We observe that $k$ must satisfy the following inequality due to the positive definiteness of the energy spectrum

$$
k \geq\left|\tilde{g}+\frac{1}{2}\right|-\frac{1}{2} .
$$

In Fig. 1, we give the diagram for $k$ versus $\tilde{g}$. Each horizontal solid line denotes the allowed values of $\tilde{g}$ for a given angular momentum $k$. For a given value of $\tilde{g}$ in this range, the energy spectrum is given by the vertical intersections with $k=$ constant lines. The circular dots at the end of each horizontal line represent the supersymmetric vacuum state, and for these particular values of $\tilde{g}$, supersymmetry is unbroken. Observe that there exists a reflection symmetry in the parameter space of the monopole charge; a given value of $\tilde{g}$ yields the same energy with $\tilde{g}^{\prime}=-\tilde{g}-1$. The spectrum is symmetric with respect to $\tilde{g}=-\frac{1}{2}$ axis. Supersymmetry is spontaneously broken unless the minimum values of $k$ reside on the lines $k=\tilde{g}$ or $k=-(\tilde{g}+1)$ in Fig. 1. In other words, the breaking occurs unless the parameter $\gamma$ is quantized, $\gamma=n / 2$ for some integers $n$.

Let us examine some cases. For symmetric ordering with the value of $(\alpha, \beta, \gamma)=\left(\frac{1}{2}, \frac{1}{2}, 0\right)$, the energy spectrum is given by

$$
E=\frac{1}{2}[k(k+1)-g(g+1)]
$$

With the substitution $k=n+g(n=0,1,2, \cdots)$, the above spectrum (4.5) agrees with the previous calculations based on the method using the shape-invariance [12]. The complete spectrum in Ref. [12] also gives the same multiplicities as our result. We observe that supersymmetry is unbroken due to the Dirac quantization condition $g=n / 2$ in this case. For asymmetric ordering with value of $(\alpha, \beta, \gamma)=\left(-\frac{1}{2}, \frac{3}{2},-\frac{1}{2}\right)$, we have

$$
E=\frac{1}{2}\left[k(k+1)-g^{2}+\frac{1}{4}\right] .
$$


Similar relation appeared in Ref. [1] where the Casimir invariant in the right hand side of Eq. (4.6) is associated with the hidden supersymmetry which is generated by the Killing-Yano tensor [19] in the $N=1$ superspace approach.

\section{SUMMARY AND DISCUSSIONS}

In summary, we investigated $N=2$ supersymmetric quantum mechanics of a charged particle on sphere in the background of magnetic monopole. Our formulation has a couple of novel aspects. First, we introduced gauged chiral superfield which is annihilated by gauge covariant superderivatives. These gauge covariant superderivatives and their associated supercharges fulfill the usual relations of supersymmetry in Eq. (2.7). We also adopted $C P(1)$ model approach which admits a compact $N=2$ superspace formulation of the problem. Then, we carried out the Dirac quantization and computed the exact quantum mechanical spectrum. We found that the spontaneous breaking of supersymmetry occurs unless the parameter $\gamma$ is quantized.

The spontaneous breaking of supersymmetry occurs in this system for generic values of $\gamma$. Recall that the parameter $\tilde{g}$ which characterizes the breaking is composed of two factors; the monopole charge $g$ and the parameter $\gamma$ representing the effect of the operator ordering ambiguity. Even in the case without monopole, spontaneous supersymmetry breaking occurs except for the case where $\gamma=n / 2$. On the other hand, the monopole effect can be dominant in the large $g$ case in which the background space becomes fuzzy sphere [20]. It would be interesting to explore the connection between the fuzzy sphere and supersymmetry further in the present framework.

\section{Acknowledgments}

We would like to thank Choonkyu Lee and Jin-Ho Cho for useful discussions. STH would like to acknowledge financial support in part from the Korea Science and Engineering Foundation Grant R01-2000-00015. The work of PO was supported by the Korea Research Foundation Grant R05-2004-000-10682-0.

\section{APPENDIX A: CLASSICAL EQUATIONS OF MOTIONS}

In order to derive the classical equations of motion, we consider the $N=2$ SUSY Lagrangian given by

$$
\begin{aligned}
L & =2|\dot{z}-(\bar{z} \cdot \dot{z}) z|^{2}+\frac{i}{2}(\bar{\psi} \cdot \dot{\psi}-\dot{\bar{\psi}} \cdot \psi)-\frac{i}{2}(\bar{z} \cdot \dot{z}-\dot{\bar{z}} \cdot z) \bar{\psi} \cdot \psi+i g(\bar{z} \cdot \dot{z}-\dot{\bar{z}} \cdot z-i \bar{\psi} \cdot \psi) \\
& +N(\bar{z} \cdot z-1)+\Lambda \bar{z} \cdot \psi+\bar{\psi} \cdot z \bar{\Lambda}
\end{aligned}
$$

where $N, \Lambda$ and $\bar{\Lambda}$ are the Lagrange multipliers associated with the second class constraints derived from (2.15). Variations of the Lagrangian A.1 over the variables $z$ and $\psi$ produce their equations of motion

$$
\begin{aligned}
D_{t} p & =-\frac{i}{4}(\bar{\psi} \cdot \psi+2 g) p-\frac{1}{8}(\bar{\psi} \cdot \psi+2 g)^{2} \bar{z}+N \bar{z}-\bar{\Lambda} \bar{\psi} \\
D_{t} \psi & =\frac{i}{2} \psi(\bar{\psi} \cdot \psi+2 g)+i z \bar{\Lambda}
\end{aligned}
$$

where the Lagrangian multipliers are given by

$$
\begin{aligned}
N & =D_{t} p \cdot z+\frac{i}{4}(\bar{\psi} \cdot \psi+2 g) p \cdot z+\frac{1}{8}(\bar{\psi} \cdot \psi+2 g)^{2}, \\
\Lambda & =-\frac{i}{2} \bar{\psi} \cdot \bar{p}, \\
\bar{\Lambda} & =\frac{i}{2} p \cdot \psi .
\end{aligned}
$$

The equations of motion for $\bar{z}$ and $\bar{\psi}$ can be readily read off from the Hermitian conjugates of the above corresponding equations. 


\section{APPENDIX B: QUANTUM COMMUTATORS}

Using the commutators Eq. (3.7), we find that

$$
\left[K_{a}, X\right]=-\frac{\sigma_{a}}{2} X, \quad X=(z, \bar{p}, \psi),
$$

and

$$
\left[K_{a}, \bar{X}\right]=\bar{X} \frac{\sigma_{a}}{2}, \quad \bar{X}=(\bar{z}, p, \bar{\psi}) .
$$

Note that these relations hold independent of $\gamma$ and confirm that $K_{a}$ 's are indeed generators of rotations. We also have

$$
\left[K_{a}, Q\right]=\left[K_{a}, \bar{Q}\right]=\left[K_{a}, \bar{\psi} \cdot \psi\right]=0 .
$$

In terms of unit vector $\vec{x}=\bar{z} \vec{\sigma} z$, the angular momentum generator $\vec{K} \equiv K_{a}$ is given by

$$
\vec{K}=\vec{x} \times \dot{\vec{x}}+(g+\gamma+1-\bar{\psi} \cdot \psi) \vec{x}
$$

Note that besides $(\gamma+1) \vec{x}$ term, this expression is the same as the well-known angular momentum in $\mathbf{R}^{3}$ in the bosonic case [3].

[1] P. A. M. Dirac, Quantized singularities in the electromagnetic field, Proc. Roy. Soc. Lon. A 133, 60 (1931).

[2] S. R. Coleman, The Magnetic Monopole Fifty Years Later in Les Houches Sum. School, 461 (1981); P. Goddard and D. I. Olive, New developments in the theory of magnetic monopoles, Rep. Prog. Phys. 41, 1357 (1978).

[3] R. Jackiw, Dynamical symmetry of the magnetic monopole, Ann. Phys. 129, 183 (1980).

[4] E. D'Hoker and L. Vinet, Supersymmetry of the Pauli equation in the presence of a magnetic monopole, Phys. Lett. B 137, 72 (1984); D. Spector, $N=0$ supersymmetry and the nonrelativistic monopole, Phys. Lett. B 474, 331 (2000), hep-th/0001008 M. S. Plyushchay, On the nature of of fermion monopole supersymmetry, Phys. Lett. B 485, 187 (2000), hep-th/0005122 V. Borokhov, A. Kapustin and X. Wu, Topological disorder operators in three-dimensional conformal field theory, JHEP 0211, 049 (2002), hep-th/0206054

[5] C. Leiva and M. S. Plyushchay, Nonlinear superconformal symmetry of a fermion in the field of a Dirac monopole, Phys. Lett. B 582, 135 (2004), hep-th/0311150 G. Papadopoulos, Conformal and superconformal mechanics, Class. Quantum Grav. 17, 3715 (2000), hep-th/0002007, and references therein.

[6] For a review, see for instance F. Cooper, A. Khare and U. Sukhatme, Supersymmetry and Quantum Mechanics, Phys. Rept. 251, 267 (1995), hep-th/9405029

[7] A. C. Davis, A. J. Macfarlane, and J. W. van Holten, The quantum mechanical supersymmetric O(3) and O(2,1) models, Nucl. Phys. B 216, 493 (1983); A. C. Davis, A. J. Macfarlane, P. C. Popat and J. W. van Holten, The quantum mechanics of the supersymmetric nonlinear sigma model, J. Phys. A 17, 2945 (1984); V. P. Akulov and A. I. Pashnev, Supersymmetric quantum mechanics and spontaneous breaking of supersymmetry at the quantum level, Theor. Math. Phys. 65, 1027 (1985); R. A. Coles and G. Papadopoulos, The geometry of the one-dimensional supersymmetric nonlinear sigma models, Class. Quantum Grav. 7, 427 (1990).

[8] B. Zumino, Supersymmetry and Kähler manifolds, Phys. Lett. B 87, 203 (1978).

[9] V. P. Akulov and A. I. Pashnev, Supersymmetric quantum mechanics and spontaneous breaking of supersymmetry at the quantum level, Theor. Math. Phys. 65, 1027 (1983); P. Salomonson and J. W. van Holten, Fermionic coordinates and supersymmetry in quantum mechanics, Nucl. Phys. B 196, 509 (1982); T. E. Clark, S. T. Love and S. R. Nowling, The supercharge and superconformal symmetry for $N=1$ supersymmetric quantum mechanics, Nucl. Phys. B 632, 3 (2002), hep-th/0108243 and reference therein.

[10] E. D'Hoker and L. Vinet, Superspace formulation of the dynamical symmetries of the Dirac magnetic monopole, Lett. Math. Phys. 8, 439 (1984).

[11] F. De Jonghe, A. J. Macfarlane, K. Peeters, and J. W. van Holten, New supersymmetry of the monopole, Phys. Lett. B 359, 114 (1995), hep-th/9507046

[12] S. Kim and C. Lee, Supersymmetry-based approach to quantum particle dynamics on a curved surface with non-zero magnetic field, Ann. Phys. 296, 390 (2002), hep-th/0112120

[13] V. de Alfaro, S. Fubini, G. Furlan and M. Roncadelli, Operator ordering and supersymmetry, Nucl. Phys. B 296, 402 (1988); M. Claudson and M. B. Halperin, Supersymmetric ground state wave funcations, Nucl. Phys. B 250, 689 (1985).

[14] A. D'Adda, P. Di Vecchia and M. Lüscher, Confinement and chiral symmetry breaking in CP $P^{N-1}$ models with quarks, Nucl. Phys. B 152, 125 (1979), and references therein; E. Witten, Supersymmetric form of the nonlinear $\sigma$-model in two dimensions, Phys. Rev. D 16, 2991 (1977). 
[15] A. P. Balachandran, G. Marmo and A. Stern, Magnetic monopoles with no strings, Nucl. Phys. B 162, 385 (1980); A. P. Balachandran, G. Marmo, B. S. Skagerstam and A. Stern Supersymmetric point particles and monopoles with no strings, Nucl. Phys. B 164, 427 (1980); Erratum-ibid. B 169, 547 (1980).

[16] T. T. Wu and C. N. Yang, Dirac's monopole without strings: classical Lagrangian theory, Phys. Rev. 14, 437 (1976).

[17] L. H. Ryder, Dirac monopoles and the Hopf map $S^{3} \rightarrow S^{2}$, J. Phys. A 13, 437 (1980); I. J. R. Aitchison, Berry phases, magnetic monopoles and Wess-Zumino terms or how the Skyrmion got its spin, Acta. Phys. Polon. B 18, 207 (1987), and references therein.

[18] C. Han, Canonical quantization of the CP(1) model with the Chern-Simons term, Phys. Rev. D 47, 5521 (1993).

[19] G. W. Gibbons, R. H. Rietdijk and J. W. van Holten, SUSY in the sky, Nucl. Phys. B 404, 42 (1993), hep-th/9303112 and the references therein.

[20] J. Madore, The fuzzy sphere, Class. Quantum Grav. 9, 69 (1992); H. Grosse, C. Klimčik and P. Prešnajder, Topologically nontrivial field configurations in noncommutative geometry, Commun. Math. Phys. 178, 507 (1996), hep-th/9510083 A. Y. Alekseev, A. Recknagel and V. Schomerus, Non-commutative world-volume geometries: branes on SU(2) and fuzzy spheres, JHEP 9909, 023 (1999), hep-th/9908040 A. P. Balachandran, B. P. Dolan, J. Lee, X. Martin and D. O'Connor, Fuzzy complex projective spaces and their star-products, J. Geom. Phys. 43, 184 (2002), hep-th/0107099 M. Hatsuda, S. Iso and H. Umetsu, Noncommutative superspace, supermatrix and lowest Landau level, Nucl. Phys. B 671, 271 (2003), hep-th/0306251 K. Hasebe and Y. Kimura, Fuzzy supersphere and supermonopole, Nucl. Phys. B 709, 94 (2005), hep-th/0409230 